B. FRUNEAU J.-P. RUDANT

Laboratoire des géomatériaux Institut francillen des géosciences Université

de Marne-la-Vallée

5, bd Descartes 77454 Marne-la-Vallée Cedex 2

fruneau@umiv-mlv.fr rudant@univ-mlv.fr

B. DEFFONTAINES

École pratique des hautes études

15. bd de la Mer 35800 Dinard deffontaines@ephe sorbonne.fr

\section{A.-M. PRUNIER-}

\section{LEPARMENTIER}

Inspection générale des carrières

Direction de la voirie et des déplacements

Mairie de Paris

1. place Denfert-Rochereau 75014 Paris

Anne-Marie.

Leparmentier@mairieparis.fi

\section{Suivi des déplacements} verticaux par interférométrie radar : exemple de la gare Haussmann-Saint-Lazare (Paris)

Une série d'interférogrammes construits à partir d'images radar ERS-1 et ERS-2 concernant les environs de la gare Saint-Lazare (Paris) nous a permis de mesurer lextension spatiale et lamplitude des déformations de surface liées aux pompages nécessaires lors des travaux de réalisation $\alpha$ hors-eau $)$ de la gare souterraine Haussmann-Saint-Lazare du RER E, et d'examiner leur évolution temporelle. Trois phases sont mises en évidence : (1) une subsidence de $1.7 \mathrm{~cm}$ au début des pompages (de fin 1993 à mi-1995), (2) une phase de stabilité durant les pompages (entre 1995 et mi-1998), enfin (3) une phase de remontée des terrains $(1,6 \mathrm{~cm})$ due à l'arrêt des pompages (à partir de mi-1998). Ces résultats concordent avec les mesures enregistrées par des piézomètres situés autour des pompages ainsi que des données de comparaison de nivellement et des données de terrain.

Mots-clés : interférométrie radar, pompage, déplacement topographique vertical, grand chantier souterrain, Paris.

\section{Monitoring vertical displacement with SAR interferometry: the Haussmann-Saint-Lazare case example}

Numerous interferograms were established from SAR images acquired by ERS- 1 and ERS-2 satellites on the Saint-Lazare area (Paris city). They are used to determine the spatial extension and the amplitude of the vertical deformations linked to the compulsory water pumping done in order to built in safety conditions the underground Haussman-Saint-Lazare Eole railway station. Three different phases were then highlighted: (1) a maximum of $1.7 \mathrm{~cm}$ subsidence after the beginning of the water pumping (from the end of 1993 to mid 1995), (2) a stability phase during the water pumping (between 1995 and mid 1998), at last (3) an uplift phase $(1.6 \mathrm{~cm})$ after the end of the water pumping (from mid 1998). These results are coherent with the numerous geological and geotechnical data deduced from the monitoring of the water tables, or the levelling, and the field work done in that area.

Key words : radar interferometry, water-pumping, vertical topographic displacement, large underground works. Paris. 


\section{1}

\section{Introduction}

Il est bien connu que les pompages en eau sont à l'origine de déformations verticales des terrains superficiels sus-jacents sur quelques centaines de mètres carrés d'extension spatiale. De tels pompages sont nécessaires dans les villes pour la mise hors d'eau de chantiers, pour les caves et parkings inondés, pour les lignes souterraines, les sous-sols des « Grands Maga-


mations verticales ne sont cependant pas facilement détectables avec les méthodes d'auscultation classiques (nivellement, géologiques, géotechniques...): leur coût est important, elles nécessitent des analyses comparatives, ne s'adaptent pas toujours très bien aux obstacles urbains et ne donnent que des résultats locaux.

La nouvelle technique d'interférométrie différentielle radar permet d'éviter certains de ces problèmes. Elle permet de détecter et mesurer de faibles déformations de surface, et d'obtenir ainsi des champs de déformations continus. L'interférométrie différentielle est utilisée depuis de nombreuses années pour un grand nombre d'applications géophysiques (Massonnet et Feigl, 1998), incluant des subsidences de terrains, et en particulier, plus récemment, des subsidences en zone urbaine (Fielding et al., 1998; Amelung et al., 1999; Fruneau et Sarti, 2000; Tesauro et al., 2000; Hoffmann et al., 2001). Cette méthode requiert I'utilisation d'images de radar à synthèse d'ouverture acquises sur une même zone à différentes dates, qui sont combinées par deux pour construire des cartes de déformations. L'avantage d'étudier de larges zones urbaines comme Paris est que la cohérence y est préservée sur de longues durées, permettant ainsi de surveiller des mouvements lents. Le problème principal rencontré dans les villes est dû aux variations de conditions atmosphériques entre les deux acquisitions d'images utilisées pour former un interférogramme, ce qui peut introduire des artefacts important sur l'interférogramme résultant (Goldstein, 1995; Zebker et al., 1997; Hanssen et al., 1999). Un calcul de corrélation d'interférogrammes indépendants peut cependant être utilisé et permet parfois d'éviter cette difficulté (Fruneau et Sarti, 2000).

Nous nous focalisons ici sur la ville de Paris, en particulier sur la zone de la gare Saint-Lazare. Ce secteur a fait l'objet de pompages, nécessaires lors des travaux de réalisation chors-eau » de la gare souterraine Haussmann-Saint-Lazare du RER E, et qui ont entrainé un faible tassement des terrains sus-jacents. Notre précédente êtude nous avait permis de détecter une zone de subsidence près de la station (Fruneau et Sarti, 2000). Nous montrons ici que l'interférométrie différentielle radar permet de suivre l'évolution temporelle des déplacements verticaux sur cette zone, et permet de distinguer trois phases principales, à savoir une subsidence, suivie d'une phase de stabilité, puis une remontée, mouvements directement liés à l'activité des pompages sur cette zone, La remontée des terrains a également été observée par interférométrie par Le Mouélic (Le Mouélic et al., 2002) et Fruneau (Fruneau et al., 2002a et b).

Après une brève présentation géologique, hydrogéologique et géotechnique de la zone d'étude, nous décrirons les résultats obtenus à partir des différents interférogrammes, résultats qui sont en accord avec les mesures piézométriques fournies par les piézomètres de ITnspection Générale des Carrières et de la SNCF, situés autour de la zone de pompages.

\section{2}

\section{Cadre géologique, géotechnique et hydrogéologique de la ville de Paris}

\section{1}

\section{Contexte géographique, géologique et hydrogéologique}

La colonne lithologique affectée par les pompages souterrains est composée de dépôts sédimentaires Eocène et actuels, décrits ci-dessous de bas en haut. Tout d'abord les sables Yprésiens sont caractérisés par un aquifère d'une forte perméabilité uniforme (Diffre, 1969; Leparmentier, 1988). Les argiles de Laon et la base indurée des calcaires grossiers assurent une transition faiblement perméable avec les calcaires grossiers lutétiens perméables en grand qui contiennent la nappe lutétienne, Au-dessus, les formations faiblement perméables des marnes et caillasses consistent en une alternance de minces couches de calcaires et de marnes où localement, dans le Nord de Paris, d'importants bancs de gypse sont visibles (de 2 à 20 m d'épaisseur). Ces derniers ont des épaisseurs très variables dépendant de plusieurs processus: les conditions de dépôts, l'altération, les percolations et l'érosion post-dépôt. Les sables de Beauchamp, comme leur nom ne le montre pas, sont des sables très argileux à la base et, au milieu de leur épaisseur, forment un écran argileux de faible perméabilité, qui sert de mur à la nappe bartonienne. Le calcaire de Saint-Ouen sus-jacent correspond à un calcaire bartonien syn-tectonique lépaisseur de $1,5 \mathrm{~m}$ sur l'anticlinal de Meudon et proche de $18 \mathrm{~m}$ dans le synclinal de Seine (fosse de Saint-Denis, au nord de Paris). Comme le Lutétien, le calcaire de Saint-Ouen présente surtout une perméabilité de fracture. Finalement, le sommet de la colonne litho-stratigraphique est composé d'une couverture d'alluvions anciennes $(2-3 \mathrm{~m}$ d'épaisseur) à moderne $(2-3 \mathrm{~m})$, de colluvions et de remblais (environ $5 \mathrm{~m}$ d'épaisseur). La nappe alluviale est hétérogène et en liaison directe mais lointaine avec la Seine. La présence d'un aquifère multicouche et de phénomènes karstiques liés à la présence de gypse et de carbonates a des effets importants sur les travaux géotechniques à Paris.

\section{2}

\section{Caractéristiques structurales des terrains}

La ville de Paris est localisée à l'intersection des deux directions structurales varisques majeures (NWSE et NE-SW) qui affectent à la fois le socle et favorisent la présence de plis de faibles longueurs d'onde dans la couverture sus-jacente (Prunier Leparmentier, 1988; Obert et al., 1997). Ces plis correspondent à la réactivation d'anciennes structures hercyniennes lors des épisodes compressifs alpins et pyrénéens et de l'extension ouest européenne Oligocène. Si les plis majeurs sont 


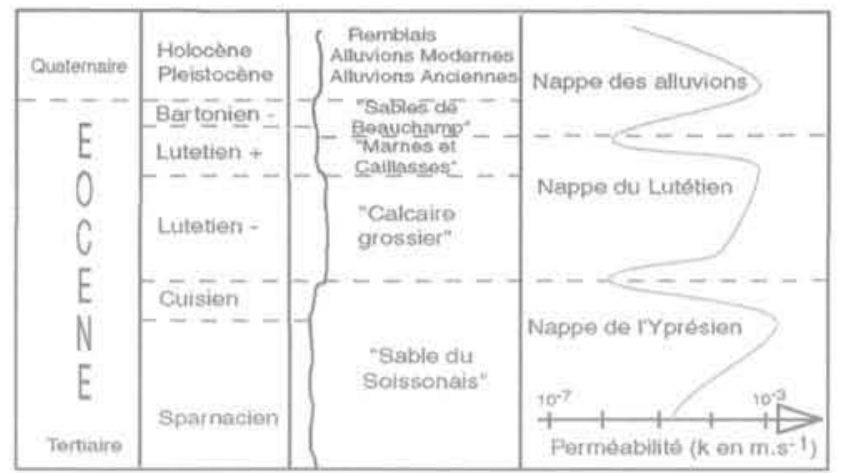

FIG 1. Colonne lithostratigraphique et perméabilité des terrains au NE de Paris (modifiés d'après Diffre, 1969 et Prunier- Leparmentier, 1988). Remarquez les aquifères lutétiens et yprésiens dans lesquels sont effectués les pompages pour construire la gare souterraine.

orientés NW-SE et présentent une amplitude d'une centaine de mètres (par exemple le sommet du $\alpha$ calcaire grossier ») est à $80 \mathrm{~m}$ NGF à l'axe de l'anticlinal de Meudon alors qu'il est à $-30 \mathrm{~m}$ NGF dans la fosse de Saint-Denis), ils sont « gauffrés ») suivant des directions E-W à N-S. Des fractures d'extrados et de nombreuses failles décrochantes conjuguées et normales affectent ces plissements (Gely et al., 1986). Par conséquent les environs de Paris présentent un réseau de plis complexes appelé parfois structure en boîte à œuf.

\section{3}

\section{Caractéristiques de site urbain}

Les Grands Magasins, l’Opéra (palais Garnier), et les parkings souterrains effectuent des pompages quotidiens afin d'éviter les entrées d'eau qui affectent l'allure générale de la nappe phréatique et des autres nappes superficielles. Par ailleurs, la réalisation d'importants ouvrages souterrains nécessite la mise hors eau du chantier durant la période des travaux (par ex. : 14 mois pour la construction du centre PTT Murat, et plus longtemps pour la construction de l'Opéra Bastille...).

Ce papier se focalise sur les variations topographiques induites par la construction de la station Haussmann-Saint-Lazare du RER E. Les pompages importants ont débuté en 1993, ont été stables pendant les travaux, puis ont diminué mi-1997 pour s'arrêter définitivement mi-1998: Nous ne discuterons pas ici des procédures de relaxation et des effets possibles de la karstification de gypse due à ces pompages, mais établirons l'extension spatiale et l'amplitude des déformations, afin de montrer l'opérationnalité de la méthode interférométrique pour le suivi de chantier en zone urbaine.

\section{Interférométrie radar}

Nous avons utilisé des images radar acquises par les satellites ERS-1 et ERS-2 entre 1993 et 1999, et avons construit plus de quarante interférogrammes, La contribution topographique dans ces différents interférogrammes a été éliminée à l'aide d'un MNT au pas de $25 \mathrm{~m}$ fourni par l'IGN. Les couples interféromé- triques ont été sélectionnés en fonction de leur ligne de base perpendiculaire, qui doit être aussi faible que possible pour éviter les effets topographiques résiduels, ainsi que la perte de cohérence géométrique : tous ces couples ont une excellente ligne de base (inférieure à $100 \mathrm{~m}$ ). Les paires ont également été choisies selon la période qu'elles couvrent, ceci afin d'examiner les différentes caractéristiques temporelles des déplacements.

Les acquisitions d'ERS-1 et ERS-2 permettent une bonne couverture temporelle des travaux de construction, excepté durant la période de subsidence: aucune image n'est disponible entre novembre 1993 et avril 1995, lorsque les pompages débutent, il est donc impossible de détecter avec précision le début du phénomène de subsidence.

Une des limitations principales de la méthode interférométrique provient de la variation des conditions atmosphériques entre les acquisitions d'images, qui peuvent introduire de larges variations de phase dans les interférogrammes résultants, et peuvent être faussement interprétées comme de la déformation. Nous avons montré dans une précédente étude que la corrélation d'interférogrammes indépendants permettait d'éviter ce problème (Fruneau et Sarti, 2000). Nous devons choisir deux paires d'interférogrammes couvrant une même période fou contenant les mêmes déplacements), mais n'ayant aucune date d'acquisition commune. En les corrélant, on peut alors séparer les artefacts atmosphériques de la déformation: seule la déformation, commune aux deux interférogrammes donnera un taux de corrélation important, tandis que les artefacts atmosphériques, en supposant que l'atmosphère soit statistiquement aléatoire d'une date à l'autre, ne donneront que des valeurs faibles de corrélation.

Grâce au nombre important d'images disponibles sur Paris, nous avons ici la possibilité d'éliminer les couples avec des artefacts troposphériques importants, facilement identifiables par leurs motifs caractéristiques, et ne conserver que ceux contenant peu d'artefacts. Pour ces interférogrammes, nous n'avons donc pas besoin d'effectuer des corrélations. Par ailleurs, nous avons la possibilité de réduire les distorsions atmosphériques en additionnant les interférogrammes indépendants, en en faisant la moyenne. Le bruit sur la phase est alors réduit d'un facteur $\sqrt{N}, N$ étant le nombre d'interférogrammes sommés (Williams et al., 1998).

\section{4}

\section{Résultats: déplacements détectés sur la zone de pompages}

Les interférogrammes différentiels ont été calculés pour quatre périodes. La figure 2 donne un exemple de quatre interférogrammes différentiels correspondant à ces périodes. Le premier (Fig. 2a) correspond à la période de stabilité du sol parisien, avant que les pompages ne commencent: aucun signal significatif ne peut être observé. Sur le second (Fig. 2b), on peut facilement détecter une tâche bleue localisée près de la gare SaintLazare. L'interférogramme correspondant à la période de pompage et donc pendant les travaux de constructions d'Eole (Fig. 2c) ne montre à nouveau aucune variation de phase, confirmant ainsi qu'aucune déformation de surface n'a eu lieu durant cette période. Le dernier (Fig. $2 \mathrm{~d})$ révèle une tache rouge, à l'endroit même où 

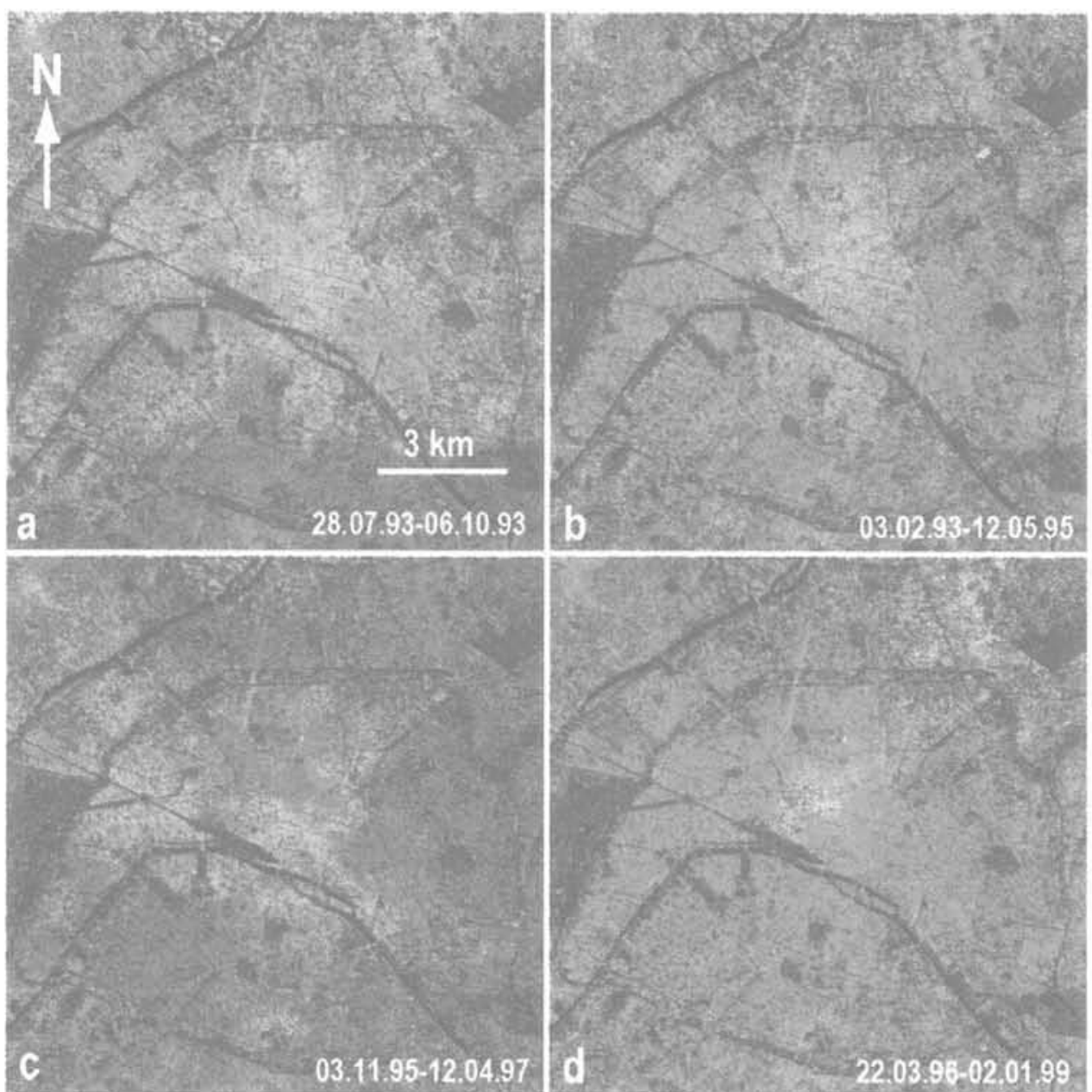

\section{remontée

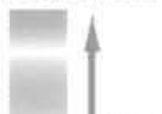

$3.1 \mathrm{~cm}$

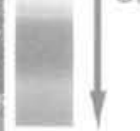

affaissement

F1G2 Interférogrammes différentiels couvrant quatre périodes différentes. Les dates des images radar sont indiquées. Un cycle complet de couleur correspond à $3,1 \mathrm{~cm}$ de déplacement vertical. (a) Aucune déformation n'est visible avant les travaux de constructions, (b) Interférogramme différentiel couvrant la période 1993-1995 faisant apparaître des déformations au début des travaux. (c) Interférogramme différentiel montrant une période de stabilité durant les pompages et les travaux. (d) Interférogramme différentiel montrant une remontée due à la fin des travaux et la lente recharge des nappes.

nous avions observé la tache bleue, et indique cette fois une remontée du sol aux environs de la gare Haussmann-Saint-Lazare, dont la construction est achevée. Le sens de variation de la phase (une augmentation ou une diminution vers le centre de la zone concernée) nous renseigne sur le type de déformation : on peut ainsi discriminer une subsidence d'une remontée.

Nous avons construit un interférogramme représentant le déplacement vertical total de la subsidence en faisant la somme de quatre interférogrammes indépendants qui présentaient déjà des artefacts atmosphériques de faible amplitude, ceci afin de réduire le niveau de bruit atmosphérique. Celui-ci est donné en figure $3 a$. La mème opération est effectuée pour la remontée: la figure $3 \mathrm{~b}$ présente des franges associées cette fois à la surrection verticale de la surface pendant la recharge en eau.

L'étendue spatiale et l'ampleur de ces phénomènes peuvent être déterminées grâce aux cartes de déplacements qui ont été construites. Dans chacun des cas, les quatre interférogrammes ont été déroulés, en convertissant l'information de phase en déplacement vertical,
Chaque frange (cycle complet de couleur) dans un interférogramme différentiel représente en effet $28 \mathrm{~mm}$ de déplacement le long de la ligne de visée du radar, soit $31 \mathrm{~mm}$ de déplacement vertical (nous faisons ici l'hypothèse que les déplacements sont purement verticaux).

Concernant l'étendue spatiale, on peut remarquer que la subsidence couvre une zone d'environ $800 \mathrm{~m}$ de large sur $1200 \mathrm{~m}$ de long, alors que la remontée concerne une zone un peu plus importante de $1800 \mathrm{~m}$ sur $2500 \mathrm{~m}$. La forme générale de la zone de déformation est une ellipse d'axe WSW-ENE. La zone de déformation maximale est formée de deux pseudos ellipses plus particulièrement visibles sur la carte de remontée: I'une est allongée, orientée NNW-SSE. L'autre, plus petite, est située plus à l'est.

L'amplitude des déplacements peut être obtenue à partír de différents profils. Différentes sections ont étè considérées pour la subsidence et la remontée, dans la mesure où la forme de la déformation n'est pas exactement la même pour les deux. L'amplitude maximale atteinte pour la subsidence est de $1,7 \mathrm{~cm}$, elle est de l'ordre de 1,6 cm pour la remontée. La comparaison de ces amplitudes nous montre que le 


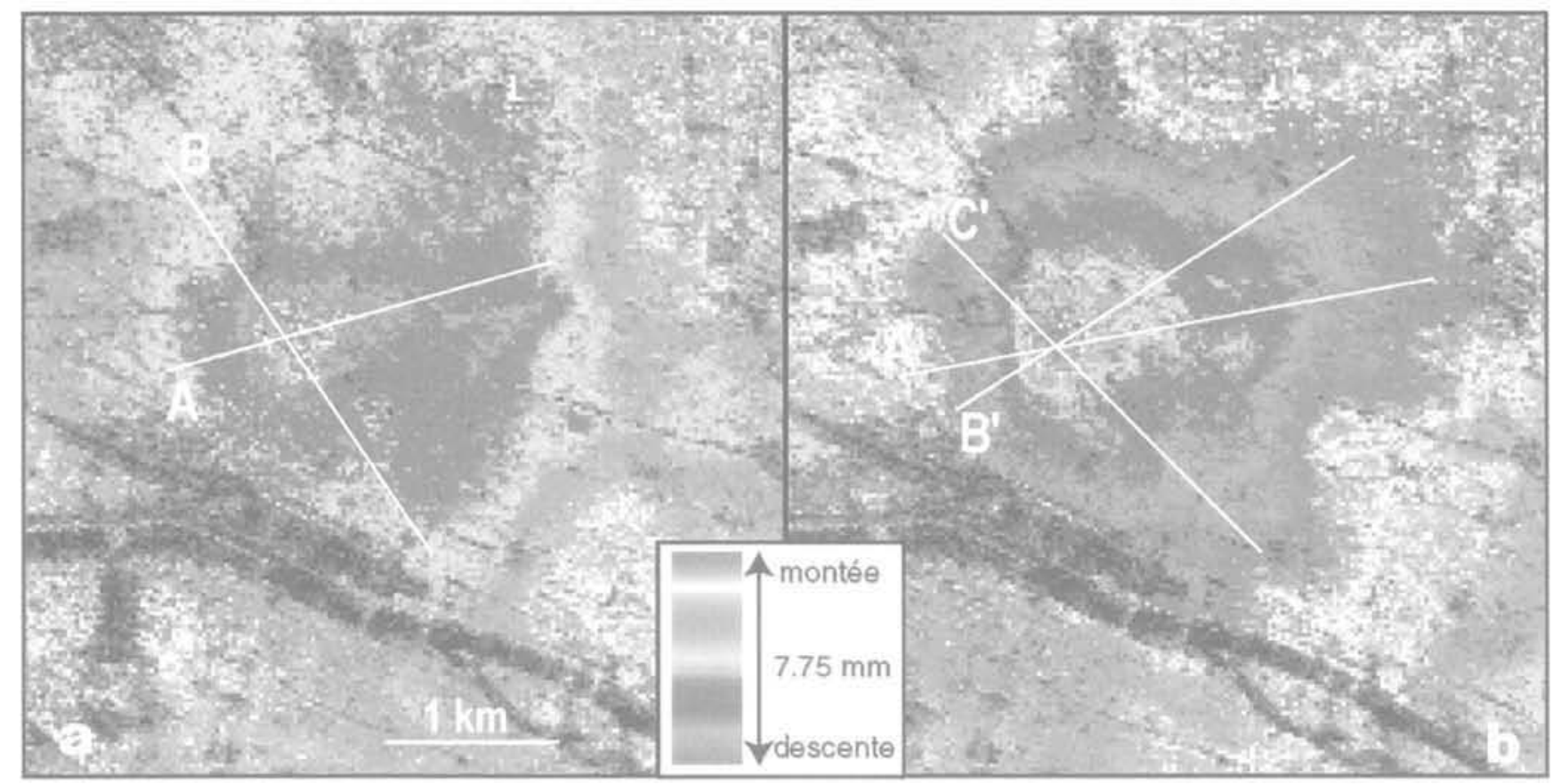

FG 3. (a) Somme de quatre interférogrammes pour la subsidence: 03.02.93-12.05.95, 06.10.93-22.07.95 28.07.93-10.08.96, et 10.03.93-16.05.95. (b) Somme de quatre interférogrammes pour la remontée 22.03.96-02.01.99, 16.02.96-17.04.99, 14.09.96-13.03.99, et 01.02.97-26.06.99. Une frange correspond ici à $7,75 \mathrm{~mm}(3,1 \mathrm{~cm} / 4)$ de déplacement vertical. Les liqnes blanches indiquent la position des profils dessinés en figure 5 .

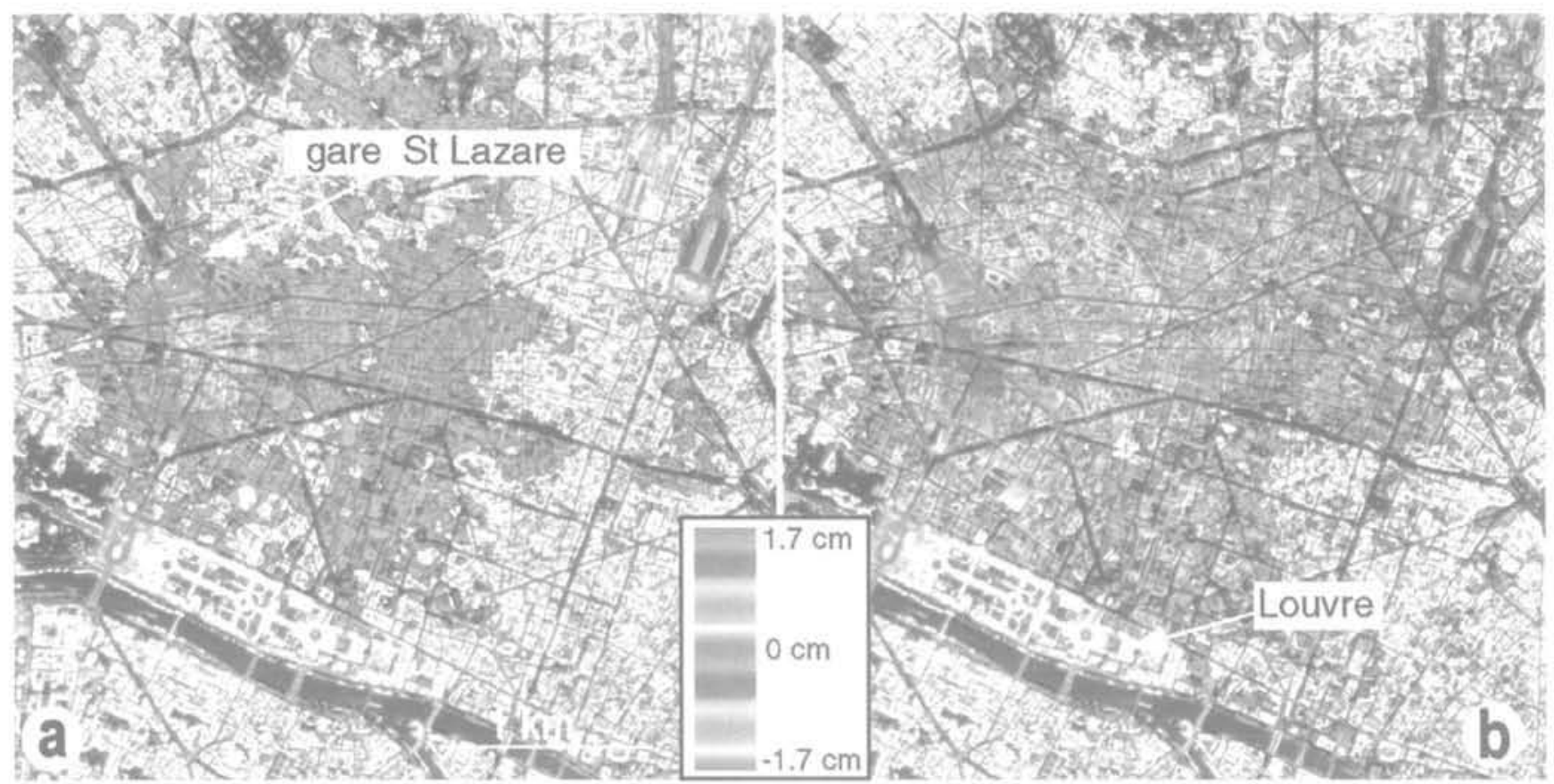

FIG 4. Carte de subsidence totale due au début des pompages en eau. Composition Intensité Teinte Saturation utilisant une photo aérienne de Paris de I'IGN (OIGN Paris 2001. Poster Paris ISSN BD Ortho'). (b) Carte de la remontée totale due à la fin des pompages et la lente recharge de la nappe (même composition ITS).

phénomène de remontée du sol compense celui de la subsidence, et montre ainsi la réversibilité de ces déformations.

Comme cela a été mentionné précédemment, aucune analyse précise de la subsidence ne peut être menée, en raison de l'absence de données en 1994

En revanche, concernant la période de remontée, nous pouvons examiner, dans la mesure du possible, mois par mois l'évolution des déformations liées à la recharge en eau, en utilisant tous les couples disponibles durant la période juillet 1997-novembre 1999.

Il apparait que la remontée s'effectue en deux phases, avec des zones différentes atteintes successivement. Malgré la présence de bulles aisément attribuables aux perturbations atmosphériques, l'interférogramme mai 1996-novembre 1997 ne montre qu'un très 

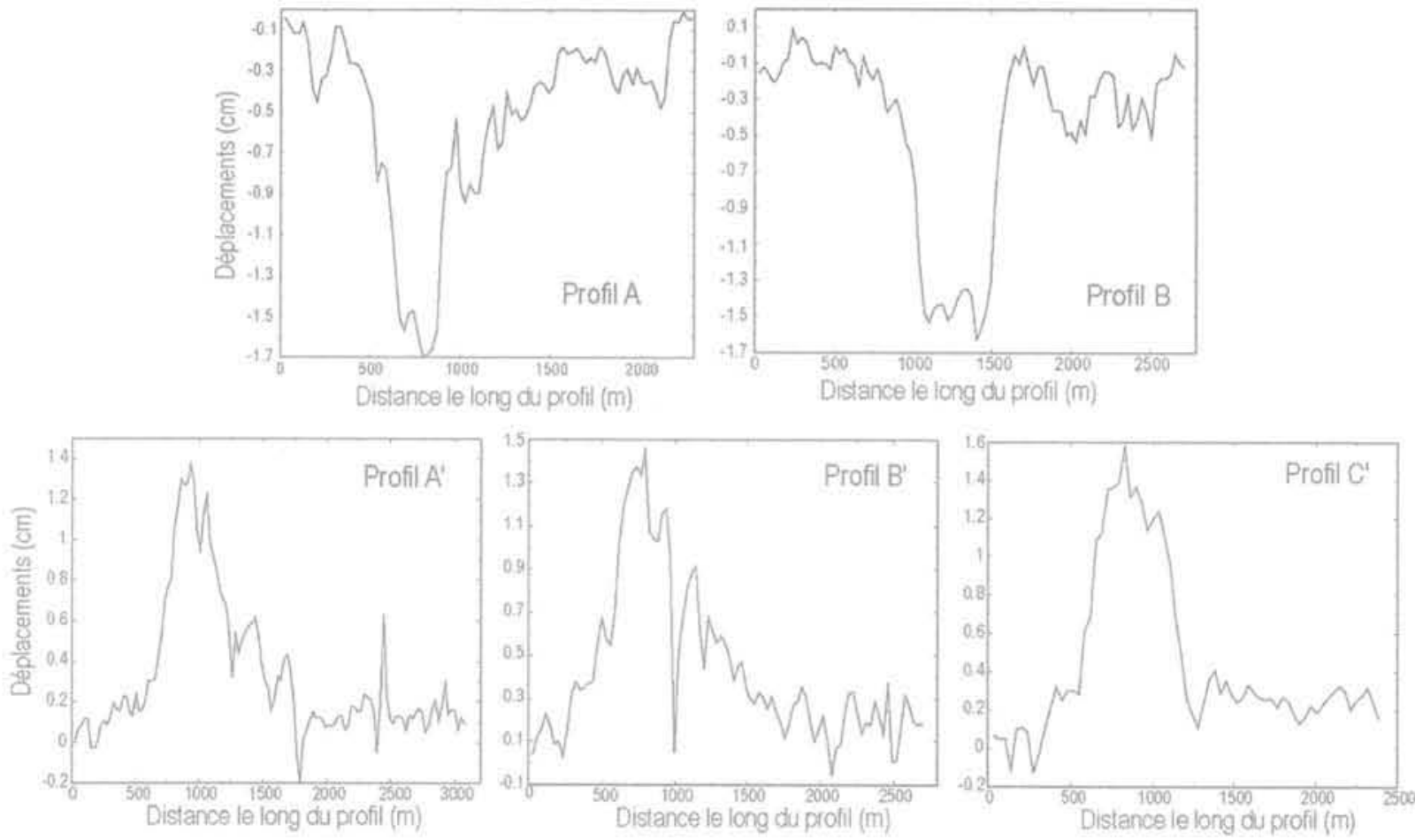

765. Tracé des différents profils (A et $B$ pour la subsidence, et $A^{\prime}, B^{\prime}$, et $C^{\prime}$ pour la remontée, voir localisation précise en figure 3 ).

faible signal sur la zone d'intérêt, signal visible à partir de juillet 1997 (interférogramme janvier 1996-juillet 1997). Un signal significatif apparaît sur l'interférogramme de septembre 1996-janvier 1998, et croit sur celui de juillet 1995-février 1998: cela indique que la remontée a débuté entre novembre 1997 et janvier 1998, ce qui est cohérent avec les mesures piézométriques. L'étendue de ces déformations demeure modeste, et localisée au-dessus de la zone de pompages. Ce signal de déformation semble stable jusqu'en juillet 1998, puis se propage vers le sud-ouest: on observe une remontée rapide, entre juillet 1998 et août 1998 d'une zone centrée approximativement à l'intersection de la rue Tronchet et de la rue des Mathurins, et qui dessine la figure géométrique sous-jacente à la remontée, à savoir une ellipse allongée orientée NNW-SSE. La surface de remontée augmente encore ensuite faiblement jusqu'en octobre 1998: sur l'interférogramme janvier 1996 octobre 1998 l'extension des déplacements semble avoir atteint sa valeur finale, elle correspond à celle observée précédemment en figure $4 \mathrm{~b}$.

\section{Piézométrie}

Le suivi de 87 piézomètres entre novembre 1990 et juin 2000 permet de mettre en évidence les fluctuations des surfaces des nappes des alluvions modernes, du Lutétien et de l'Yprésien pendant toute la durée des travaux. Bien que les pompages SNCF aient été effectués dans la nappe du Cuisien (Yprésien), nous ne disposons que de quatre valeurs de piézomètres du Cuisien. 10 Compte tenu de l'importancé du réseau de piézomètres du lutétien et de l'effet de drainance entre les nappes du Lutétien et de l'Yprésien, l'étude piézométrique est essentiellement effectuée sur le lutétien.

Le graphique de suivi (Fig. 6) indique que la nappe des alluvions ne connaît aucune variation remarquable, cette nappe ne serait pas affectée par les pompages. Au contraire, les nappes du lutétien et de l'Yprésien accusent une très forte baisse (supérieure à - $20 \mathrm{~m}$ ) en mai 1994 et inversement une très forte hausse, d'amplitude similaire, entre mars et juillet 1998 .

On peut ainsi définir plusieurs périodes:

- mai 1992-mai 1994: trois petites variations semi-réversibles de la surface piézométrique

- mai 1994-juin 1994: période de brutale et forte baisse de la surface piézométrique

- juin 1994-mars 1998: période de stabilité;

- mars 1998-septembre 1998: période de brutale et forte remontée;

- septembre 1998-juin 2000: période de stabilité.

Ces variations sont d'origine anthropique : les variations observées en juillet 1991, juin 1992 et avril 1993 correspondent à des essais de pompages, et les périodes de brusques variations correspondent aux périodes de début et arrêt des pompages inhérents au chantier afin d'abaisser la nappe pour effectuer le gros ceuvre à sec.

On note que ces différentes périodes de variations (brusques variations, et stabilité entre juin 1994 et mars 1998) sont sensiblement identiques avec celles observées par interférométrie. Les variations de la piézométrie expliquent donc bien les modifications de la 


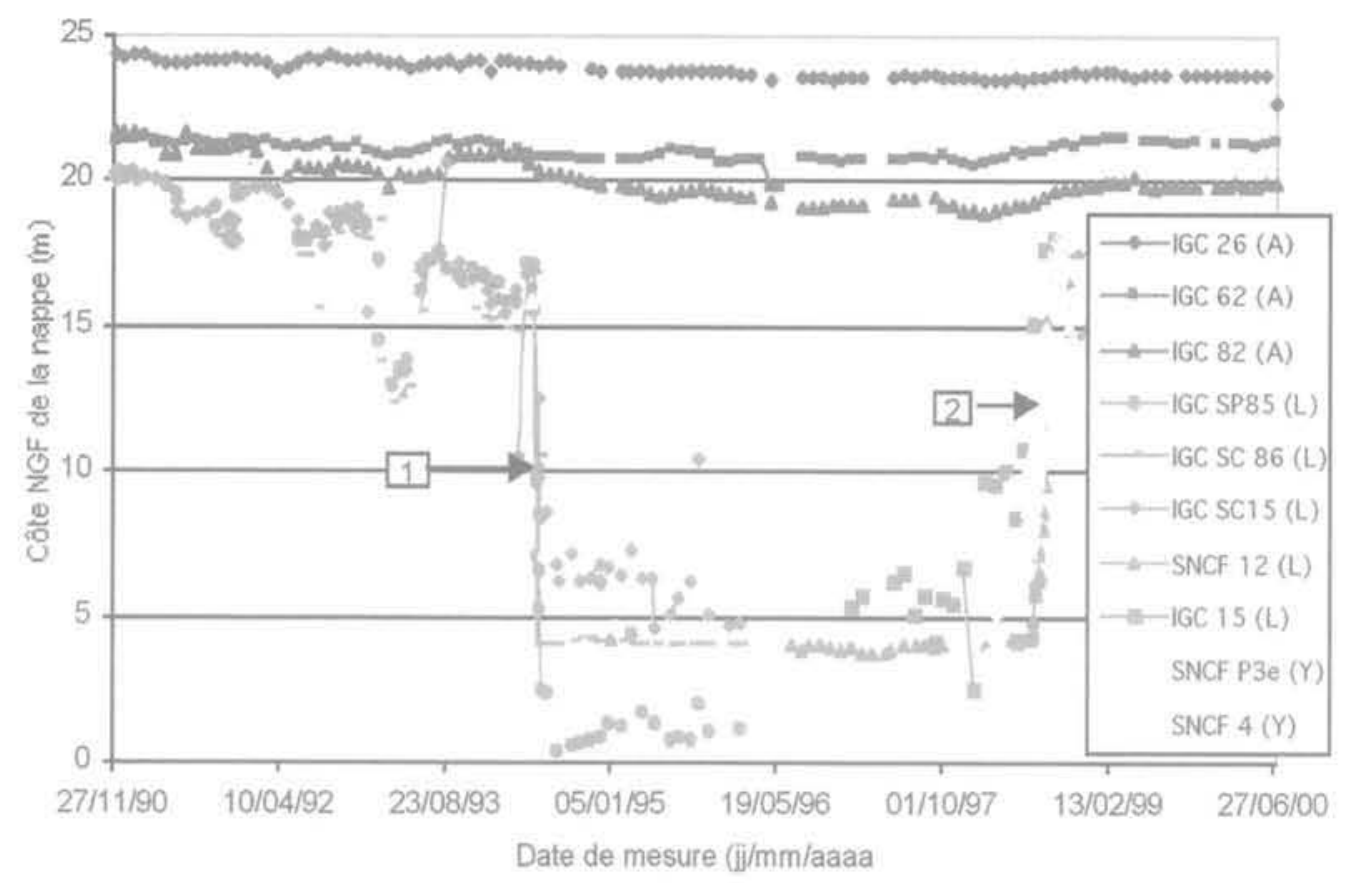

FG6. Suivi piézométrique des aquifères des $8^{e}$ et $9^{e}$ arrondissements : alluvions modernes, Lutétien et Yprésien de novembre 1990 à juin 2000. A : alluvions, L: Lutétien, Y: Yprésien. 1: mai-juin 1994, 2: juin-août 1998.

topographie dont le champ de " déformation » est cartographiable par interférométrie radar.

\section{6}

\section{Conclusion}

Grâce à l'analyse d'une série d'interférogrammes, nous sommes capables d'enregistrer des faibles déformations du sol dans la ville de Paris dans les deux sens, affaissement et remontée. Nous sommes en mesure de séparer les différentes phases de construction souterraine de la zone Haussmann-St-Lazare-Condorcet entreprise pour le RER E, spécifiquement avant, pendant et après les pompages nécessaires à la mise horseau du chantier.

Trois phases sont mises en évidence: 1) une subsidence au début des pompages (de fin 1993 à mi-1995); 2) une phase de stabilité durant les pompages (entre 1995 et fin 1997); enfin 3) une phase de remontée des terrains due à l'arrêt des pompages (à partir de mi1998). Cette remontée se fait en trois temps: une légère remontée, suivie d'une forte augmentation entre juillet 1998 et août 1998, puis une lente variation jusqu'en novembre 1998. Aucune variation significative n'est enregistrée sur la période avant et après le chantier (1993-1998), ce qui suggère que la surface topographique est revenue à son altitude initiale.
Afin de valider notre approche, nous avons utilisé des mesures piézométriques de I'IGC et de la SNCF, qui confirment les fluctuations des nappes du Lutétien et de I'Yprésien dues aux pompages pendant la période de construction.

Quelques questions subsistent, notamment en ce qui concerne le champ de déplacement observé par interférométrie. Il est oblique à l'orientation est-ouest de la gare et n'est pas centré sur la zone de pompage. Diverses hypothèses sur la géométrie structurale ainsi que l'influence d'autres pompages sous-estimés (station Auber, ligne Météor...) sont évoquées.

L'approche combinée d'interférogrammes, de données géologiques, géophysiques et hydrogéologiques permet de mieux contraindre le champ de déformation engendré par les grands travaux (cartographie et mesure des déplacements) et démontre ainsi l'opérationnalité de cette méthode en contexte urbain.

\section{REMERCIEMENTS}

Cette étude a été menée dans le cadre d'un projet soutenu par le Réseau Terre et Espace (ministère de la Recherche et CNES). Nous remercions I'ESA et le CNES qui nous ont fourni les images radar, ainsi que I'TGN pour le MNT. Nous exprimons nos plus vifs remerciements à I'TGC ainsi qu'à la SNCF, qui a manifesté son intérét pour nos recherches en nous apportant un concours financier et en alimentant nos hypothèses de ses propres données recueillies pendant la construction du RER E. Merci également à D. Obert. 
Amelung F. Galloway D. Bell J.. Zebker H. Laczniak R. - Sensing the ups and downs of Las Vegas: InSAR reveals structural control of land subsidence and aquifer-system deformation. Geologv, 27, 6. 1999, p. 483-486

Diffre P, - Géologie dynamique - Hydrologie de Paris et sa banlieue. Thèse de doc torat, Paris, 1969, $345 \mathrm{p}$.

Flelding E., Blom R., Goldstein R, - Rapid subsidence over oil fields measured by SAR interferometry. Geophys, Res, Lett., 25, 1998, p. 3215-3218

Fruneats B., Sarti F. - Detection of ground subsidence on the city of Paris using radar interferometry: isolation of deformation from atmospheric artifacts using correlation. Geophys, Res. Lett., 27, 2000, D. $3981-3984$

Fruneau B. Deffontaines B. Rudant J.P. Etude des déformations de surface sur la ville de Paris par interférométrle diffé. rentielle satellitaire, Rap. SNCF-UMLV. $n^{\circ}$ 2. juin 2002 (a), $20 \mathrm{p}$.

Fruneau B., Deffontaines B. Rudant J.P. Mesure de mouvements verticaux de surface en contexte urbain par interférométrie radar. SITEF 2002, RTE, Espace et Société, Innovations et perspectives, $23-26$ octobre 2002 (b), Toulouse.
Geiy J.P. Mercier S. Obert D. - Plis croisés et fracturation des calcaires lutétiens aux alentours du faubourg SaintJacques (Paris), Bull. Soc: Géol. France. 23, 4. 1986, p. 11-17.

Goldstein R. - Atmospheric limitations to repeat-track radar interferometry. Geophys. Res. Lett. 22, 1995, p. 2517. 2520 .

Hanssen R.F., Weckwerth T.M. Zebker H.A. Klees R. - High-resolution water vapor mapping from interferometric radar measurements. Science, 283. 1999 , p. $1297-1299$.

Hoffman J., Zebker H.A. Galloway D.L. Amelung F. - Seasonal subsidence and rebound in Las Vegas Valley, Neyada, observed by synthetic aperture radar interferometry. Water Resour. Res., 37 2001. p. 1551-1566.

Le Mouélic S., Raucoules D., Carnec C. King C - A ground uplift in the city of Paris (Erance) detected by satellite radar interferometry, Geophys. Res. Lett., 29, 2002, p. 1853-1856

Massonnet D., Feigl K. - Radar interferometry and its application to changes in the Earth's surface. Rev. Geophys., 36, 4, 1998, p. 441-500
Obert D.. Deffontaines B. Gely J.P. Joly M. Lacombe O, Lorenz J., Pomerol C. - Tectonique plicative de couverture dans le Lutétien supérieur de la vallée de l'Eure: indice d'un accident profond. Bull. Inf. Géol. Bass. Paris, 34, 2, 1997, p. 13-18.

Prunier-Leparmentier A.M. - Les problèmes géologiques et géotechniques de la ville de Paris. Thèse de doctorat de I'École nationale supérieure des mines de Paris, 1998, 244 p.

Tesauro M. Berardino P. Lanari R.. Sansosti E., Fornaro F. - Urban subsidence inside the city of Napoli (italy) observed by satellite radar interferometry. Geophys. Res. Lett., 27. 2000, p. 1961-1964.

Williams S., Bock Y., Peng F. - Integrated satellite interferometry: Tropospheric noise, GPS estimates and implications for interferometric synthetic aperture radar products, I. Geophys. Res. 103 1998, p. 27051-27067.

Zebker H.A., Rosen P.A., Hensley S, Atmospheric effects in interferometric synthetic aperture radar surface deformation and topographic maps. J. Geophys. Res., 102, 1997, p. $7547-$ 7563. 